\title{
Single intravitreal injection of etamsylate for the treatment of geographic atrophy associated with submacular hemorrhage
}

\begin{abstract}
Geographic atrophy of the retinal pigment epithelium is a devastating complication of age-related macular degeneration. We show that a single intravitreal injection of etamsylate reduces geographic atrophy and clears submacular hemorrhage in a patient with dry age-related macular degeneration. This improvement was associated with a significant gain of visual acuity.
\end{abstract}

Keywords: geographic atrophy, inflammation, submacular hemorrhage, intravitreal etamsylate, fibroblast growth factor

\author{
Volume 7 Issue I - 2017 \\ Cuevas Pedro,' Outeiriño Luis, ${ }^{2}$ Azanza \\ Carlos, ${ }^{2}$ Angulo-Frutos Javier, ${ }^{3}$ Cuevas- \\ Bourdier Adrián, ${ }^{4}$ Giménez-Gallego \\ Guillermo 5 \\ 'Universidad Alfonso X el Sabio, Spain \\ 2Departamento de Oftalmología, Hospital de Día Pío XII, Spain \\ ${ }^{3}$ Departamento de Investigación, Hospital Universitario Ramón \\ y Cajal, Spain \\ ${ }^{4}$ Laboratoire National de Santé, Dudelange, Luxembourg \\ ${ }^{5}$ Departamento de Estructura y Función de Proteínas, Centro \\ de Investigaciones Biológicas, Spain
}

Correspondence: Cuevas Pedro, Facultad de Medicina, Universidad Alfonso X, Madrid,

Email pedro.cuevas44@gmail.com

Received: October 23, 2016 | Published: July 05, 2017

\section{Introduction}

Age-related macular degeneration (AMD) is the major worldwide cause of blindness in the elderly. It is characterized by a progressive loss of color and fine vision, reduced contrast, and spatiotemporal sensitivity. The two principal types of AMD are wet or exudative and dry AMD. Wet AMD occurs when blood vessels grow through disturbed Bruch's membrane and extend from the choroids in a biological process called choroidal neovascularization (CNV). The primary treatment of $\mathrm{CNV}$ is anti-vascular endothelial growth factor (VEGF) injections. This therapy has been used to inhibit CNV and protect the retina against leakage. However, the vast majority of AMD patients have the currently untreatable dry or atrophic form of AMD, which is characterized by inflammation-driven degeneration of the retinal pigment epithelium (RPE). ${ }^{1}$ Geographic atrophy (GA) is an advanced form of dry AMD characterized by the loss of an area of the RPE that grows over time. ${ }^{2}$ In this case report we show the efficacy of a single intravitreal injection of etamsylate, an anti-inflammatory drug, ${ }^{3}$ in a patient with GA associated submacular hemorrhage.

\section{Case presentation}

This study was performed with patient's consent after the Ethical Committee approval of our Institution. A 66 years old women presented with hypertension and a three months history of progressive visual loss in her left eye. Ocular examination including Snellen visual acuity (VA) measurements and color fundus photography was performed at baseline and one and three months after treatment. Color fundus photography shows macular edema and features of dry AMD with a submacular hemorrhage that masks a macular GA (Figure 1). GA shows the following characteristics: round or ovoid shape feature, sharp demarcation between relative healthy RPE and visibility of choroidal vessels.
The patient received an intravitreal injection $(150 \mu l)$ of etamsylate $(125 \mathrm{mg} / \mathrm{ml}$; Dicynone, Sanofi-Aventis, France) following the International Guidelines for intravitreal injections ${ }^{4}$ in her left eye. The clearance of submacular hemorrhage was observed after three months of treatment (Figure 1) concomitantly with a significant progressive reduction of GA $(70 \%)$, suggesting that etamsylate may participate in the regeneration of RPE. Clearance of submacular hemorrhage and reduction of GA was associated with a progressive gain of VA: 0.05 at baseline versus 0.08 and 0.40 after one and three months of treatment, respectively.

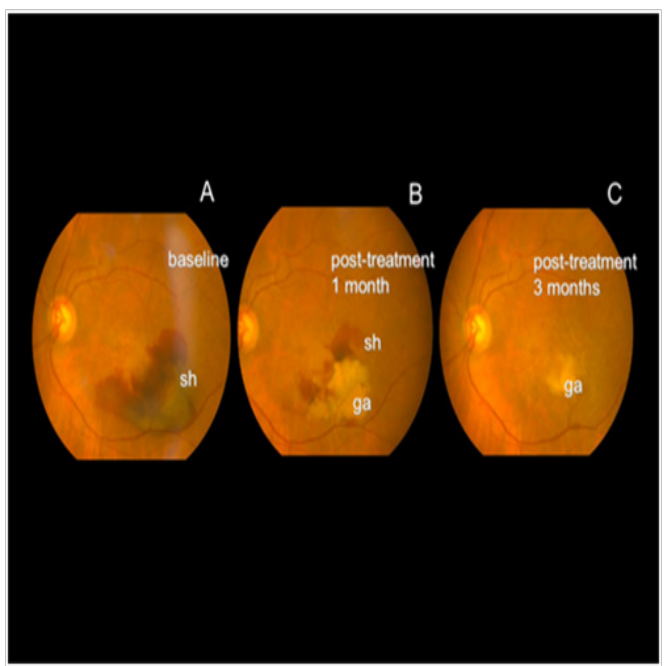

Figure I Color fundoscopy at baseline, and after one and three months of intravitreal etamsylate administration. A sharp demarcation between regions of retinal pigment epithelium loss and intact retinal pigment epithelium is seen. Within the regions of geographic atrophy (ga), choroidal vessels are seen, sh: submacular hemorrhage. 


\section{Discussion}

The retinal pigment epithelium (RPE) is a partner of the neural retina and is indispensable for vision in humans. RPE has a wide variety of function, as such nutrient supply, oxidative stress protection, phagocytosis, and secretion of survival signals for retinal cells. ${ }^{5}$ In addition, RPE has a potential for regeneration..$^{6,7}$ The sequence of events leading to $\mathrm{GA}$ is the progression of large drusen to hyperpigmentation, followed by regression of the drusen, hypopigmentation and ultimately RPE cell death, leading to atrophic area of the retina underlying choroids. ${ }^{8}$

Opposed to anti-VEFG therapy of exudative AMD, there are not any currently approved treatment for GA. Current clinical trials are investigating remedies for dry GA through multiple approaches, including inflammation, the visual cycle, neuroprotection and cell replacement therapy. ${ }^{9-12}$ Aetiology of dry AMD suggests that the treatment of inflammation could be a suitable alternative to treat dry AMD. ${ }^{13}$ Fibroblast growth factor (FGF), in spite of being an angiogenesis promoter, ${ }^{14}$ is also involved in inflammation processes $^{15-19}$ and seems to play key roles in neurodegenerative diseases such as AMD. ${ }^{20}$ It has been shown that etamsylate inhibits several FGF-related activities as such inflammation. ${ }^{21}$ Etamsylate is a synthetic haemostatic agent that has been used orally and systemically for many years. Furthermore, local delivery seems a better choice in order to reach appropriate therapeutic concentrations of etamsylate in well delimited target organs or tissues, as is the case for dry AMD.

This case report shows that a single intravitreal injection of etamsylate leads to clearance of submacular hemorrhage, resolution of macular edema, reduction of GA and vision improvement that occurs within one month and continues to improve for the next three months. It seems obvious that anti-inflammatory activities of etamsylate ${ }^{3}$ may, at least in part, contribute to a clinical improvement of GA, which was observed in the present report.

\section{Conclusion}

Intravitreal etamsylate injection shows significant improvement of visual acuity over time, associated to submacular hemorrhage clearance and diminution of the geographic atrophy in a patient with dry age-related macular degeneration.

\section{Acknowledgements}

None.

\section{Conflict of interest}

The author declares no conflict of interest.

\section{References}

1. Gehrs KM, Anderson DH, Johnson LV, et al. Age-related macular degeneration-emerging pathogenetic and therapeutic concepts. Ann Med. 2006;38(7):450-471.

2. Danis RP, Lavine JA, Domalpally A. Geographic atrophy in patients with advanced dry age-related macular degeneration:current challenges and future prospects. Clin Ophthalmol. 2015;9:2159-2174.

3. Zawrotniak M, Kozik A, Rapala-Kozik M. Selected mucolytic, anti-inflammatory and cardiovascular drugs change the ability of neutrophils to form extracellular traps (NETs). Acta Biochim Pol. 2015;62(3):465-473.

4. Aiello LP, Brucker AJ, Chang S, et al. Evolving guidelines for intravitreous injections. Retina. 2004;24(5):S3-19.
5. Strauss O. The retinal pigment epithelium in visual function. Physiol Rev. 2005;85(3):845-881

6. Han JW, Lyu J, Park YJ, et al. Wnt/ $\beta$-catenin signaling mediates regeneration of retinal pigment epithelium after laser photocoagulation in mouse eye. Invest Ophthalmol Vis Sci. 2015;56(13):8314-8324.

7. Kobayashi M, Iwese T, YamamotoK, et al. Association between photoreceptor regeneration and visual acuity following surgery for rhegmatogenous retinal detachment. Invest Ophthalmol Vis Sci. 2016;57(3):889-898.

8. Gass JD. Drusen and disciform macular detachment and degeneration. Arch Ophthalmol. 1973;90(3):206-217.

9. Patel HR, Eichenbaum D. Geographic atrophy:clinical impact and emerging treatments. Ophthalmic Surg Lasers Imaging Retina. 2015;46(1):813 .

10. Do DV, Pieramici DJ, van Lookeren Campagne M, et al. A phase ia dose-escalation study of the anti-factor D monoclonal antibody fragment FCFD4514S in patients with geographic atrophy. Retina. 2014;34(2):313-320.

11. Kubota R, Al-Fayoumi S, Mallikaarjun S, et al. Phase 1, dose-ranging study of emixustat hydrochloride (ACU-4429), a novel visual cycle modulator, in healthy volunteers. Retina. 2014;34(3):603-609.

12. Schwartz SD, Regillo CD, Lam BL, et al. Human embryonic stem cellderived retinal pigment epithelium in patients with age-related macular degeneration and Stargardt's macular dystrophy:follow-up of two openlabel phase 1/2 studies. Lancet. 2015;385:509-516.

13. Buschini E, Piras A, Nuzzi R, Vercelli A. Age related macular degeneration and drusen: neuroinflammation in the retina. Prog Neurobiol. 2011;95(1):14-25.

14. Gimenez-Gallego G, Rodkey J, Bennett C, et al. Brain-derived acidic fibroblast growth factor: complete amino acid sequence and homologies. Science. 1985;230(4732):1385-1388.

15. Byrd VM, Ballard DW, Miller GG, et al. Fibroblast growth factor-1 (FGF-1) enhances IL-2 production and nuclear translocation of NF-kappaB in FGF receptor-bearing Jurkat T cells. J Immunol. 1999;162(10):5853-5059.

16. Meij JT, Sheikh F, Jimenez SK, et al. Exacerbation of myocardial injury in transgenic mice overexpressing FGF-2 is T cell dependent. Am J Physiol Heart Circ Physiol. 2002;282(2):H547-H555.

17. Rossini M, Cheunsuchon B, Donnert E, et al. Immunolocalization of fibroblast growth factor-1 (FGF-1), its receptor (FGFR-1), and fibroblast-specific protein-1 (FSP-1) in inflammatory renal disease. Kidney Int. 2005;68(6):2621-2628.

18. Zittermann SI, Issekutz AC. Basic fibroblast growth factor (bFGF, FGF2) potentiates leukocyte recruitment to inflammation by enhancing endothelial adhesion molecule expression. Am J Pathol. 2006;168(3):835846.

19. Presta M, Andrés G, Leali D, et al. Inflammatory cells and chemokines sustain FGF2-induced angiogenesis. Eur Cytokine Netw. 2009;20(2):3950 .

20. Lee M, Kang Y, Suk K, et al. Acidic fibroblast growth factor (FGF) potentiates glial-mediated neurotoxicity by activating FGFR2 IIIb protein. J Biol Chem. 2011;286(48):41230-41245.

21. Fernández IS, Cuevas P, Angulo J, et al. Gentisic acid, a compound associated with plant defense and a metabolite of aspirin, heads a new class of in vivo fibroblast growth factor inhibitors. $J$ Biol Chem. 2010;285(15):11714-11729. 